\title{
Enhanced PID Controllers Design Based on Modified Smith Predictor Control for Unstable Process with Time Delay
}

\author{
Chengqiang Yin, Jie Gao, and Qun Sun \\ School of Mechanical and Automobile Engineering, Liaocheng University, Liaocheng 252059, China \\ Correspondence should be addressed to Chengqiang Yin; shtjycq@163.com
}

Received 1 July 2014; Revised 30 August 2014; Accepted 1 September 2014; Published 29 September 2014

Academic Editor: Hongli Dong

Copyright ( 2014 Chengqiang Yin et al. This is an open access article distributed under the Creative Commons Attribution License, which permits unrestricted use, distribution, and reproduction in any medium, provided the original work is properly cited.

\begin{abstract}
A two-degree-of-freedom control structure is proposed for a class of unstable processes with time delay based on modified Smith predictor control; the superior performance of disturbance rejection and good robust stability are gained for the system. The setpoint tracking controller is designed using the direct synthesis method; the IMC-PID controller for disturbance rejection is designed based on the internal mode control design principle. The controller for set-point response and the controller for disturbance rejection can be adjusted and optimized independently. Meanwhile, the two controllers are designed in the form of PID, which is convenient for engineering application. Finally, simulation examples demonstrate the validity of the proposed control scheme.
\end{abstract}

\section{Introduction}

Unstable processes are well known to be difficult to control especially when there exists pure time delay. A time delay is introduced into the transfer function description of such system due to the measurement delay or an actuator delay [1-5]. A lot of academic research had been devoted to developing effective control strategies for such processes. Generally, PI or PID controllers are designed using a unity feedback control structure for these systems. Two-degreeof-freedom methods based on PID control are the most common methods [6-11]. Internal mode control and Smith predictor (SP) control are regarded as the most effective methods for process control and most widely used in industry but cannot be used directly for unstable process with time delay. Owing to the standard SP control structure which is in essence equivalent to the internal model control structure for time delay processes, a number of control schemes based on modified Smith predictor had been developed in recent years [12-18]. By using the Smith predictor, Rao and Chidambaram [19] proposed a two-degree-of-freedom control scheme for unstable systems with time delay; in the scheme three controllers were used to improve the system performance. Liu et al. [20] proposed a modified form of Smith predictor in a two-degree-of-freedom control scheme, which demonstrated the remarkable improvement of regulatory capacity for both of reference input tracking and load disturbance rejection. García and Albertos [21] proposed a scheme that is equivalent to the Smith predictor but able to cope with any kind of systems; the results showed a substantial improvement in the performance/robustness tradeoff as well as in the tuning process. Vijayan and Panda [22] proposed a double-feedback loop method which was used to achieve stability and better performance of the process. By comparison, few papers [23, 24] developed discrete-time domain control methods for advanced regulation of unstable processes. Besides, nonlinear control schemes were presented to deal with integrating and unstable processes with time delay [25]. Dey et al. [26] proposed an autotuning proportional-derivative control scheme; the proportional and derivative gains were adjusted using a nonlinear gain updating factor to achieve an overall improved performance.

Evaporation processes are common in the food industry. It is the stage in which the water contained in a juice is eliminated in order to obtain a juice with a higher concentration. The dynamics of the evaporation can be represented with integrating first order plus time delay process [27]. The control of such system is difficult because of the limitations imposed by the integrator and the time delay on the system performance and stability. 


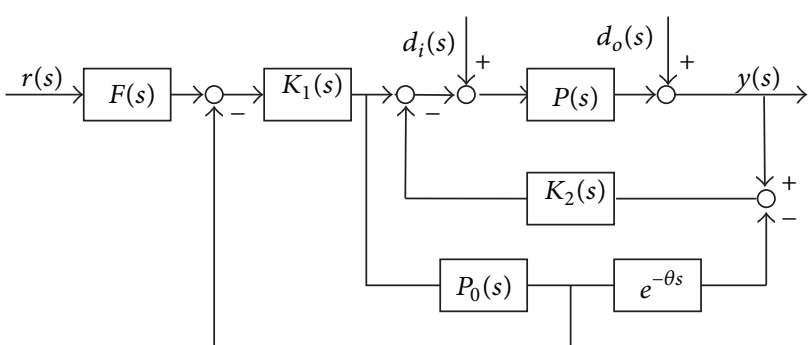

FIGURE 1: Modified Smith control structure.

Disturbance rejection is much more important than setpoint tracking for many process control applications. But the methods proposed previously for the disturbance rejection have not gained much popularity; what is more, it is difficult to be carried out in process industries. The objective of the present study is to develop a practicable method to obtain enhanced disturbance rejection performance and perfect setpoint tracking performance. So a two-degree-of-freedom control scheme based on modified Smith predictor shown in Figure 1 is proposed. The set-point tracking controller $K_{1}(s)$ and disturbance rejection controller $K_{2}(s)$ are designed in the form of PID. The scheme can lead to substantial control performance improvement, especially for the disturbance rejection. The analysis has been carried out for the two typical transfer function models: $P(s)=k e^{-\theta s} / s(T s-1)$ and $P(s)=$ $k e^{-\theta s} / s(T s+1)$.

In Figure $1 P_{0}(s)$ is the transfer function of the process model without the time delay, that is, $P(s)=P_{0}(s) e^{-\theta s}, K_{1}(s)$ is used for set-point tracking, $K_{2}(s)$ is used for disturbance rejection, $F(s)$ is the set-point filter, $r(s)$ is the set point, $y(s)$ is the process output, and $d_{i}(s)$ and $d_{o}(s)$ are the disturbances before and after process, respectively. As can be seen, the performance of set point and load disturbance rejection response are decoupled completely and can be monotonically tuned to meet a good performance by controller $K_{1}(s)$ and $K_{2}(s)$, respectively.

\section{Controller Design Procedure}

2.1. Set-Point Tracking Controller $K_{1}(s)$. From Figure 1, the transfer function from $y(s)$ to $r(s)$ can be determined in the form of

$$
\begin{aligned}
H_{r}(s) & =\frac{y(s)}{r(s)} \\
& =\frac{P(s) K_{1}(s)}{1+P_{0}(s) K_{1}(s)} \frac{1+K_{2}(s) P_{0}(s) e^{-\theta s}}{1+K_{2}(s) P(s)} .
\end{aligned}
$$

In the nominal case, that is $P(s)=P_{0}(s) e^{-\theta s}$, the set-point tracking transfer function can be simplified as

$$
H_{r}(s)=\frac{y(s)}{r(s)}=\frac{P(s) K_{1}(s)}{1+P_{0}(s) K_{1}(s)} .
$$

Obviously, there is no dead-time element in the characteristic equation of the nominal set-point tracking transfer function; $K_{1}(s)$ can be obtained if the transfer function is determined:

$$
K_{1}(s)=\frac{H_{r d}(s)}{1-H_{r d}(s)} \frac{1}{P_{0}(s)} .
$$

Considering the implementation and system performance, the desired set-point tracking transfer function is proposed:

$$
H_{r d}(s)=\frac{y(s)}{r(s)}=\frac{a_{2} s^{2}+a_{1} s+1}{(\lambda s+1)^{3}},
$$

where $\lambda$ is the adjustable parameter; as for the unstable process type $P(s)=k e^{-\theta s} / s(T s-1)$, the controller can be derived from (3) and (4):

$$
K_{1}(s)=\frac{s(T s-1)\left(a_{2} s^{2}+a_{1} s+1\right)}{k\left[(\lambda s+1)^{3}-\left(a_{2} s^{2}+a_{1} s+1\right)\right]} .
$$

Because of simple structure and better control performance than the direct-action tuner, the ability of PID controllers to meet most of the control objectives has led to their widespread acceptance in the control industry. As we know, distributed control system is widely used in process industry. PID module is the basic and the most used module in the distributed control system; over $90 \%$ control points were designed in PID form [28]. To obtain a realizable controller, $K_{1}(s)$ should be realized in discrete form or approximated by a rational transfer function. So $K_{1}(s)$ can be expressed as

$$
K_{1}(s)=\frac{(T s-1)\left(a_{2} s^{2}+a_{1} s+1\right)}{k\left[\lambda^{3} s^{2}+\left(3 \lambda^{2}-a_{2}\right) s+\left(3 \lambda-a_{1}\right)\right]} .
$$

According to the model transform method [29], order $a_{1}=$ $4 \lambda, a_{2}=6 \lambda^{2}+1$. A PID controller with first order lag filter can be approximated

$$
K_{1}(s)=k_{1}\left(1+\frac{1}{\tau_{i 1} s}+\tau_{d 1} s\right) \frac{1}{\alpha s+1} .
$$

After approximate comparison, we can obtain the parameters of PID, $k_{1}=a_{1} / k$ is proportional gain, $\tau_{i 1}=a_{1}$ is integral gain, $\tau_{d 1}=a_{2} / a_{1}$ is derivative gain, and $\alpha=\lambda^{4} / T$ is the filter parameter.

As can be seen from (4), numerator of the transfer function will result in undesired system overshoot. So in order to improve the set-point tracking performance and reduce the overshoot, a set-point filer is designed $F(s)=$ $1 /\left(a_{2} s^{2}+a_{1} s+1\right)$.

Analogously, as for the process type $P(s)=k e^{-\theta s} / s(T s+1)$, $K_{1}(s)$ can be obtained from (3) and (4):

$$
K_{1}(s)=\frac{(T s+1)\left(a_{2} s^{2}+a_{1} s+1\right)}{k\left[\lambda^{3} s^{2}+\left(3 \lambda^{2}-a_{2}\right) s+\left(3 \lambda-a_{1}\right)\right]} .
$$

Using the similar method, we obtain $a_{1}=3 \lambda, a_{2}=3 \lambda^{2}-\lambda^{3} / T$. The $K_{1}(s)$ can be derived in the form of PID as follows:

$$
K_{1}(s)=k_{1}\left(1+\frac{1}{\tau_{i 1} s}+\tau_{d 1} s\right),
$$


where $k_{1}=3 T / k \lambda^{2}$ is proportional gain, $\tau_{i 1}=3 \lambda$ is integral gain, and $\tau_{d 1}=\lambda(1-\lambda / 3 T)$ is derivative gain.

2.2. Disturbance Rejection Controller $K_{2}(s)$. In the proposed control structure shown in Figure 1, the load disturbance transfer functions are given by

$$
\begin{aligned}
& H_{d_{i}}(s)=\frac{y(s)}{d_{i}(s)}=\frac{P(s)}{1+K_{2}(s) P(s)}, \\
& H_{d_{o}}(s)=\frac{y(s)}{d_{o}(s)}=\frac{1}{1+K_{2}(s) P(s)} .
\end{aligned}
$$

At the same time, we can obtain the closed-loop complementary sensitivity function between the process input and output for the load disturbance rejection as

$$
T(s)=\frac{K_{2}(s) P(s)}{1+K_{2}(s) P(s)} .
$$

Here, $K_{2}(s)$ is designed using the method of unit feedback based on internal mode control theory [30]:

$$
\frac{K_{2}(s) P(s)}{1+K_{2}(s) P(s)}=P(s) C(s),
$$

where $C(s)$ is the internal mode controller, $P(s)=P_{-}(s) P_{+}(s)$, $C(s)=P_{-}^{-1}(s) f(s)$, in which $f(s)$ is the filter, $P_{-}(s)$ contains the invertible portion of the model, and $P_{+}(s)$ contains all the noninvertible portion. The invertible portions are the part of the model with stable poles and unstable poles. The noninvertible portions are the portion of the model with right half plane zeros and time delays. In order to ensure that the system is internally stable, the filter is designed as

$$
f(s)=\frac{\sum_{i=1}^{m} b_{i} s^{i}+1}{\left(\lambda^{\prime} s+1\right)^{n}},
$$

where $\lambda^{\prime}$ is an adjustable parameter which controls the tradeoff between the performance and robustness, determined to cancel the unstable and integrating poles of $P(s) . m$ is the number of unstable and integrating poles. $n$ is selected to be large enough to make the internal mode controller proper; $b_{i}$ is determined by $1-\left.P(s) C(s)\right|_{s=z_{1}, \ldots, z_{m}}=0$, where $z_{1}, \ldots, z_{m}$ are the unstable and integrating poles.

As for the unstable process type $P(s)=k e^{-\theta s} / s(T s-1)$, it can be transformed as $P(s)=k^{\prime} e^{-\theta s} /\left(T^{\prime} s-1\right)(T s-1)$; time constant $T^{\prime}$ is selected to be large enough. The filter is designed as

$$
f(s)=\frac{b_{2} s^{2}+b_{1} s+1}{\left(\lambda^{\prime} s+1\right)^{4}} .
$$

Correspondingly by using (12) and (14), the controller $K_{2}(s)$ can be obtained as

$$
K_{2}(s)=\frac{\left(T^{\prime} s-1\right)(T s-1)\left(b_{2} s^{2}+b_{1} s+1\right)}{k^{\prime}\left[\left(\lambda^{\prime} s+1\right)^{4}-e^{-\theta s}\left(b_{2} s^{2}+b_{1} s+1\right)\right]},
$$

where $b_{1}$ and $b_{2}$ are determined by the two constraints

$$
\begin{aligned}
\lim _{s \rightarrow 1 / T} H_{d_{0}}(s)=0, \quad \lim _{s \rightarrow 1 / T^{\prime}} H_{d_{0}}(s)=0, \text { that is, } \\
\lim _{s \rightarrow 1 / T}\left[1-\frac{b_{2} s^{2}+b_{1} s+1}{(\lambda s+1)^{4}} e^{-\theta s}\right]=0, \\
\lim _{s \rightarrow 1 / T^{\prime}}\left[1-\frac{b_{2} s^{2}+b_{1} s+1}{(\lambda s+1)^{4}} e^{-\theta s}\right]=0 .
\end{aligned}
$$

Following a simple calculation, we obtain

$$
\begin{gathered}
b_{1}=\left(T^{\prime 2}\left(\frac{\lambda^{\prime}}{T^{\prime}}+1\right)^{4} e^{\theta / T^{\prime}}-T^{2}\left(\frac{\lambda^{\prime}}{T}+1\right)^{4} e^{\theta / T}\right. \\
\left.+T^{2}-T^{\prime 2}\right) \times\left(T^{\prime}-T\right)^{-1}, \\
b_{2}=T^{\prime 2}\left[\left(\frac{\lambda^{\prime}}{T^{\prime}}+1\right)^{4} e^{\theta / T^{\prime}}-1\right]-b_{1} T^{\prime} .
\end{gathered}
$$

The dead time $e^{-\theta s}$ in (15) is approximated using Pade expansion:

$$
e^{-\theta s}=\frac{1-\theta s / 2}{1+\theta s / 2}
$$

Then substituting (18) into (15) obtains the controller $K_{2}(s)$ as

$$
K_{2}(s)=\frac{b_{2} s^{2}+b_{1} s+1}{\eta} \times \frac{\left(T^{\prime} s-1\right)(T s-1)(1+\theta s / 2)}{1+l_{1} s+l_{2} s^{2}+l_{3} s^{3}+l_{4} s^{4}},
$$

where $\eta=4 \lambda^{\prime}-b_{1}+\theta, l_{1}=\left(6 \lambda^{\prime 2}+2 \lambda^{\prime} \theta+b_{1} \theta / 2-b_{2}\right) / \eta$, $l_{2}=\left(4 \lambda^{\prime 3}+3 \lambda^{\prime 2} \theta+b_{2} \theta^{2} / 2\right) / \eta, l_{3}=\left(\lambda^{\prime 4}+2 \lambda^{\prime 3} \theta\right) / \eta$, and $l_{4}=\lambda^{\prime 4} \theta^{2} / 2 \eta$. Since the resulting controller does not have a standard PID controller form, a procedure is employed to produce a PID controller cascade with a first order lead-lag filter:

$$
K_{2}(s)=k_{2}\left(1+\frac{1}{\tau_{i 2} s}+\tau_{d 2} s\right) \frac{1+\alpha^{\prime} s}{1+\beta s} .
$$

As can be seen from (19), the first term $\left(b_{2} s^{2}+b_{1} s+1\right) / \eta$ can be interpreted as the standard PID controller in the form of $k_{2}\left(1+\left(1 / \tau_{i 2} s\right)+\tau_{d 2} s\right)$. The later term can be interpreted as a first order lead-lag filter $\left(1+\alpha^{\prime} s\right) /(1+\beta s), \alpha^{\prime}=0.5 \theta ; \beta$ can be obtained by

$$
\left.\frac{d}{d s}\left(v s^{2}+\beta s+1\right)\right|_{s=0}=\left.\frac{d}{d s}\left[\frac{1+l_{1} s+l_{2} s^{2}+l_{3} s^{3}+l_{4} s^{4}}{\left(T^{\prime} s-1\right)(T s-1)}\right]\right|_{s=0} .
$$

Since the $v s^{2}$ term has little impact on the overall control performance, it can be ignored. Calculating by using (19) and (21), we can obtain the parameters for the controller $K_{2}(s)$. $k_{2}=b_{1} / k^{\prime}\left(4 \lambda^{\prime}+\theta-b_{1}\right)$ is proportional gain, $\tau_{i 2}=b_{1}$ is 
integral gain, and $\tau_{d 2}=b_{2} / b_{1}$ is derivative gain. $\alpha^{\prime}=0.5 \theta$, $\beta=\left(\left(b_{1} \theta / 2-b_{2}+2 \lambda^{\prime} \theta+6 \lambda^{\prime 2}\right) /\left(\theta+4 \lambda^{\prime}-b_{1}\right)\right)+T+T^{\prime}, \alpha$ and $\beta$ are parameters of the lead-lag filter.

As for the process type $P(s)=k e^{-\theta s} / s(T s+1)$, it can be transformed as $P(s)=-k^{\prime} e^{-\theta s} /\left[\left(T^{\prime} s-1\right)(-T s-1)\right]$, and the executable controller $K_{2}(s)$ can be obtained in the form of PID by using (15), (18), and (20). On the basis of simulation study on integrating first order plus time delay processes, the use of $0.1 \beta$ instead of $\beta$ is suitable and $\beta$ is about $0.2-1.2$ generally.

\section{System Robust Stability Analysis}

A control system is robust if it is insensitive to differences between the actual system and the model of the system which was used to design the controller. These differences are referred to as model mismatch or simply model uncertainty [31]. A study of robustness analysis is an important task because no mathematical model of a system will be a perfect representation of the actual system. Small-gain theorem is $\left\|l_{m} T(s)\right\|_{\infty}<1$; it expresses the robustly stable condition of a control system, where $l_{m}(s)$ is the bound on the process multiplicative uncertainty and $T(s)$ is the closed-loop complementary sensitivity function [32].

For the process type $P(s)=k e^{-\theta s} / s(T s-1)$, if there are uncertainties that exist in all three parameters, that is,

$$
P^{\prime}(s)=\frac{(k+\Delta k) e^{-(\theta+\Delta \theta) s}}{s\left(T_{2} s-1\right)(\Delta T s+1)} .
$$

The bound on the process multiplicative uncertainty $l_{m}(s)$ can be obtained as

$$
l_{m}(s)=\left|\frac{P^{\prime}(s)-P(s)}{P(s)}\right|=\frac{(1+(\Delta k / k)) e^{-\Delta \theta s}}{(\Delta T s+1)}-1 .
$$

Then the tuning parameters should be selected in such a way that

$$
\left\|\frac{b_{2} s^{2}+b_{1} s+1}{\left(\lambda^{\prime} s+1\right)^{4}}\right\|_{\infty}<\frac{1}{\left\|\left((1+(\Delta k / k)) e^{-\Delta \theta s} /(\Delta T s+1)\right)-1\right\|_{\infty}} .
$$

If the uncertainty exists in the time delay, the tuning parameters should be selected in a way that

$$
\left\|\frac{b_{2} s^{2}+b_{1} s+1}{\left(\lambda^{\prime} s+1\right)^{4}}\right\|_{\infty}<\frac{1}{\left|e^{-j \Delta \theta w}-1\right|} .
$$

At the same time, in order to compromise the nominal performance with the robust stability of the closed-loop for the load disturbance rejection, the following constraint is required to meet [31]

$$
\left|l_{m}(s) T(s)\right|+|\omega(s)(1-T(s))|<1,
$$

where $\omega(s)$ is a weight function of the closed-loop sensitivity function $S=1-T(s)$, which usually can be chosen as

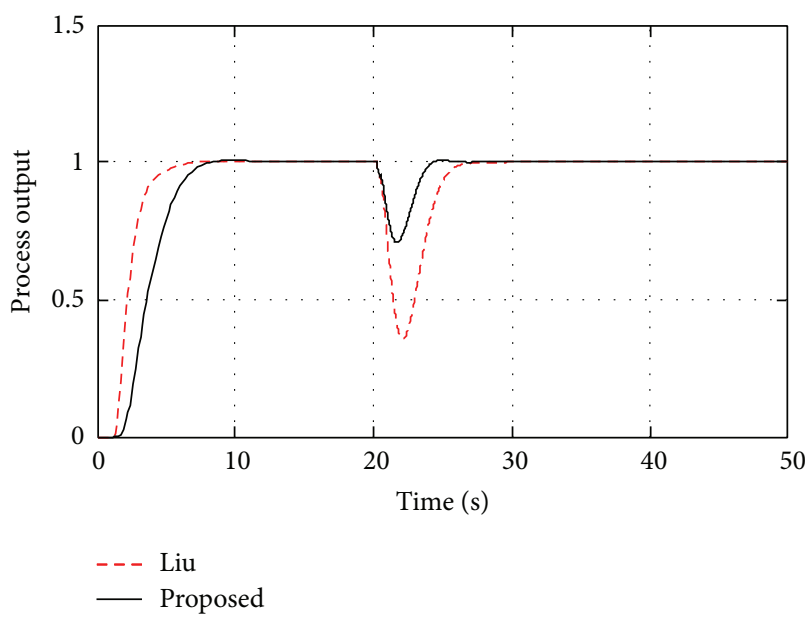

Figure 2: Nominal system responses for Example 1.

$1 / s$ for the step change of the load disturbance. It indicates that tuning the adjustable parameter $\lambda^{\prime}$ aims at the tradeoff between the nominal performance of the closed-loop and its robust stability. That is to say, decreasing $\lambda^{\prime}$ improves the disturbance rejection performance of the closed-loop but decays its robust stability in the presence of the process uncertainty. On the contrary, increasing $\lambda^{\prime}$ tends to strengthen the robust stability of the closed-loop but degrades its disturbance rejection performance.

\section{Simulation}

Example 1. Consider the unstable process studied by Liu et al. [20], $P(s)=e^{-0.2 s} / s(s-1)$.

In the proposed method, take $\lambda=3.3 \theta=0.66$, the parameters of controllers are obtained, $k_{1}=2.64, \tau_{i 1}=2.64$, $\tau_{d 1}=1.367, \alpha=0.19, a_{1}=2.64$, and $a_{2}=3.61$. As for the controller $K_{2}(s)$, transform the process as $P(s)=$ $100 e^{-0.2 s} /(100 s-1)(s-1)$, take $\lambda^{\prime}=2 \theta=0.4$, and obtain

$$
K_{2}(s)=3.02\left(1+\frac{1}{1.79 s}+1.06 s\right) \frac{0.1 s+1}{0.008 s+1} .
$$

The method proposed in [20] is better than others which can be seen in this simulation effects, so only compare with the method in [20]. By adding a unit step change to the set-point input at $t=0$, an inverse unit step change of load disturbance to process output at $t=20$, the simulation results are obtained as shown in Figure 2.

Now suppose that there exists $30 \%$ increment for estimating the process time delay and $30 \%$ reduction for the time constant of the process model; then the perturbed system responses are provided in Figure 3.

It can be seen from the simulation results that the proposed method gives better performances for the unstable process.

Example 2. Consider a process studied by Liu and Gao [15], $P(s)=0.1 e^{-5 s} / s(5 s+1)$. 


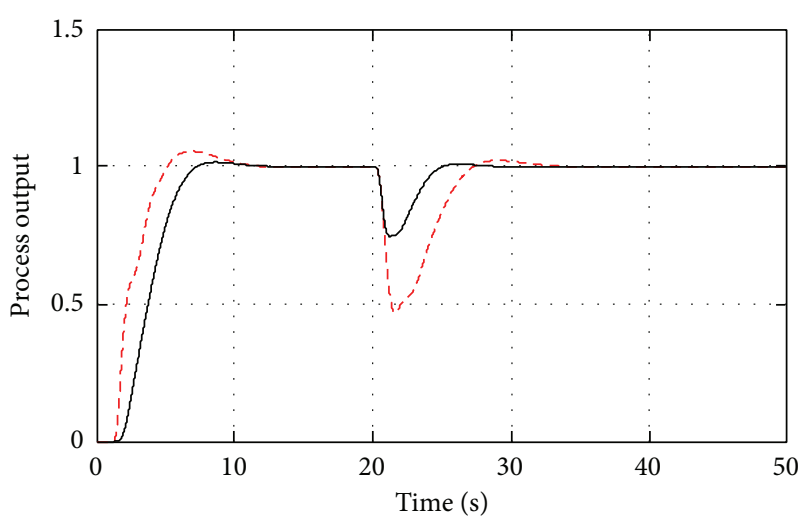

-- - Liu

- Proposed

Figure 3: Perturbed system responses for Example 1.

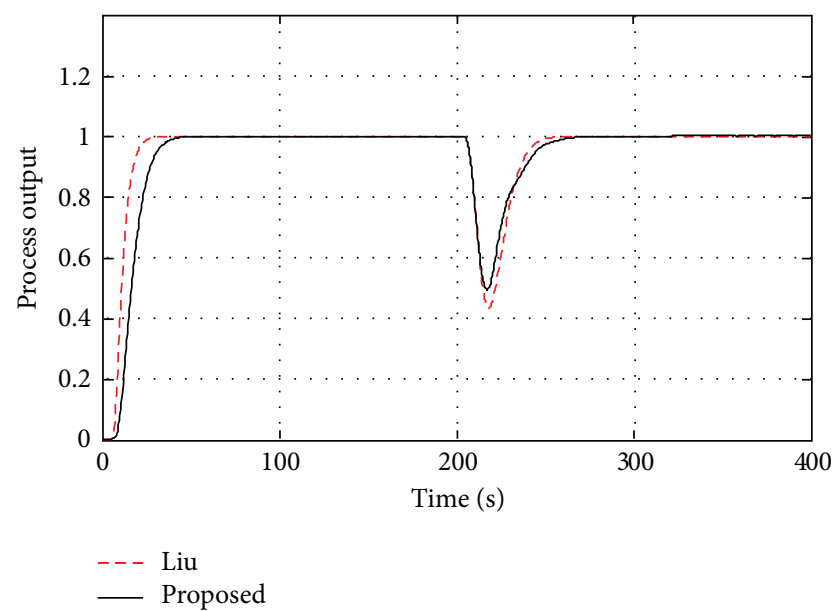

FIGURE 4: Nominal system responses for Example 2.

In the proposed method, as for controllers $K_{1}(s)$, take $\lambda=0.8 \theta=4$, and obtain $k_{1}=9.375, \tau_{i 1}=12, \tau_{d 1}=2.933$, $a_{1}=12$, and $a_{2}=35.2$. As for controller $K_{2}(s)$, transform the process as $P(s)=-10 e^{-5 s} /(100 s-1)(-5 s-1)$, take $\lambda^{\prime}=0.8 \theta=4$, and obtain the controller

$$
K_{2}(s)=2.2\left(1+\frac{1}{22 s}+3.864 s\right) \frac{2.5 s+1}{0.8 s+1}
$$

Add a unit step change to the set-point input at $t=0$ and an inverse step change of load disturbance to the process output at $t=200$. The simulation results are obtained as shown in Figure 4, and the corresponding control action responses are shown in Figure 5. It can be observed from the figure that the control action response of the proposed method shows smooth variation compared to that of Liu and Gao [15].

Now suppose that there exist $10 \%$ error for estimating the process time delay and the time constant of the process model, such as both of them are actually $10 \%$ larger. The perturbed system responses are provided in Figure 6, and the corresponding control action responses are shown in Figure 7. It can be seen that the proposed control action

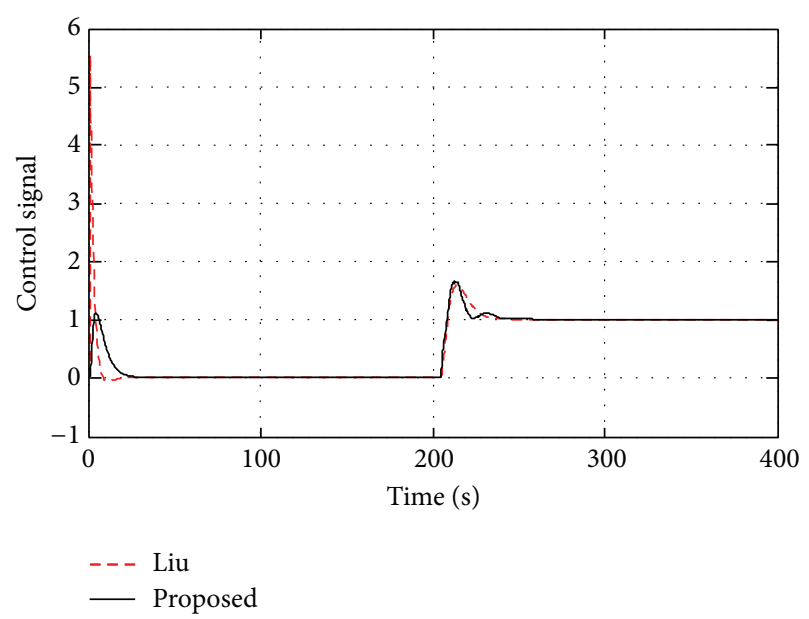

Figure 5: Nominal system control signal for Example 2.

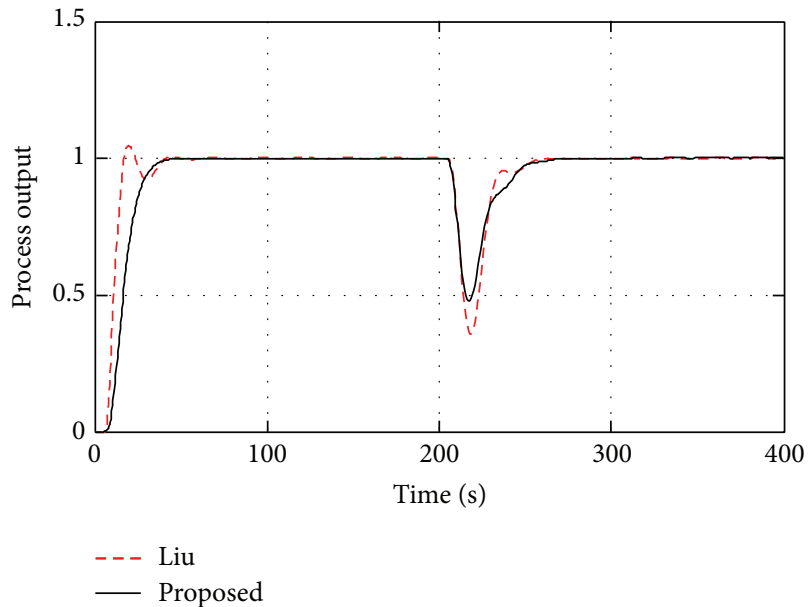

Figure 6: Perturbed system responses for Example 2.

is smooth and the performance of disturbance rejection is better than that of Liu and Gao [15].

\section{Conclusion}

In order to improve the system performance of disturbance rejection, a modified Smith predictor scheme has been proposed based on a two-degree-of-freedom control structure. In the proposed control structure, both of the setpoint response and the load disturbance response can be tuned separately by the set-point tracking controller and the disturbance estimator, respectively. The most advantage of the method is that the designed system has good performance of disturbance rejection as well as performance of set-point tracking. The two controllers are all designed in the form of PID, and they are simple and easy to be used in process industry. Comparisons with the previous methods demonstrate a clear advantage of the proposed method in both nominal and robust performances in disturbance rejection. 


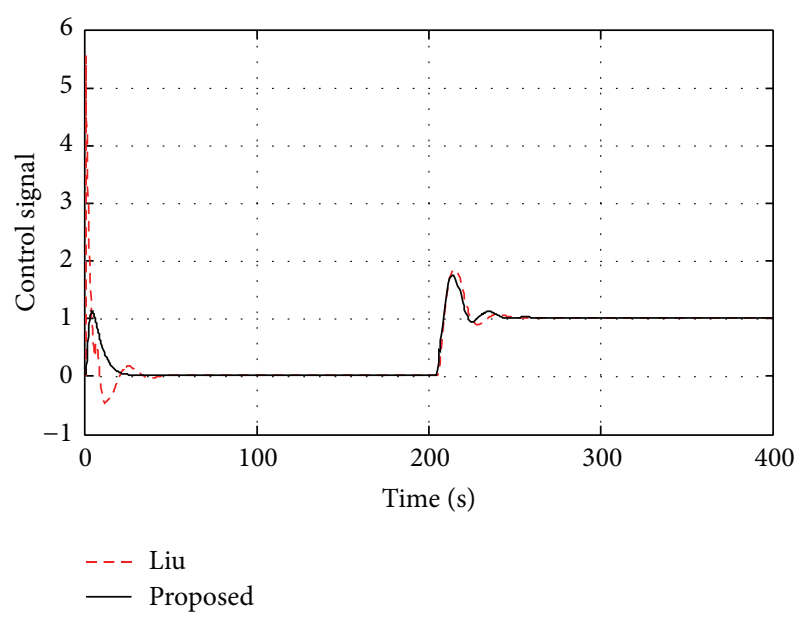

Figure 7: Perturbed system control signal for Example 2.

\section{Conflict of Interests}

The authors declare that there is no conflict of interests regarding the publication of this paper.

\section{Acknowledgments}

This research is funded by the Natural Science Foundation of Shandong Province (Grant no. ZR2012CQ026) and the Science and Technology Funds of Shandong Education Department (Grant no. J11LD16).

\section{References}

[1] I.-L. Chien, S. C. Peng, and J. H. Liu, "Simple control method for integrating processes with long deadtime," Journal of Process Control, vol. 12, no. 3, pp. 391-404, 2002.

[2] M. Huzmezan, W. A. Gough, G. A. Dumont, and S. Kovac, "Time delay integrating systems: a challenge for process control industries. A practical solution," Control Engineering Practice, vol. 10, no. 10, pp. 1153-1161, 2002.

[3] H. Dong, Z. Wang, and H. Gao, "Distributed $H_{\infty}$ filtering for a class of markovian jump nonlinear time-delay systems over lossy sensor networks," IEEE Transactions on Industrial Electronics, vol. 60, no. 10, pp. 4665-4672, 2013.

[4] J. E. Normey-Rico, C. Bordons, and E. F. Camacho, Control of Dead-Time Processes, Spinger, Berlin, Germany, 2007.

[5] Z. Wang, H. Dong, B. Shen, and H. Gao, "Finite-horizon $H_{\infty}$ filtering with missing measurements and quantization effects," IEEE Transactions on Automatic Control, vol. 58, no. 7, pp. 17071718, 2013.

[6] A. Visioli, "Optimal tuning of PID controllers for integral and unstable processes," IEE Proceedings: Control Theory and Applications, vol. 148, no. 2, pp. 180-184, 2001.

[7] C. Hwang and J. Hwang, "Stabilisation of first-order plus deadtime unstable processes using PID controllers," IEE Proceedings: Control Theory and Applications, vol. 151, no. 1, pp. 89-94, 2004.

[8] R. C. Panda, "Synthesis of PID controller for unstable and integrating processes," Chemical Engineering Science, vol. 64, no. 12, pp. 2807-2816, 2009.
[9] Y. Lee, J. Lee, and S. Park, "PID controller tuning for integrating and unstable processes with time delay," Chemical Engineering Science, vol. 55, no. 17, pp. 3481-3493, 2000.

[10] A. Seshagiri Rao, V. S. Rao, and M. Chidambaram, "Direct synthesis-based controller design for integrating processes with time delay," Journal of the Franklin Institute, vol. 346, no. 1, pp. 38-56, 2009.

[11] D. G. Padhan and S. Majhi, "A new control scheme for PID load frequency controller of single-area and multi-area power systems," ISA Transactions, vol. 52, no. 2, pp. 242-251, 2013.

[12] I. Kaya, "Autotuning of a new PI-PD smith predictor based on time domain specifications," ISA Transactions, vol. 42, no. 4, pp. 559-575, 2003.

[13] S. Majhi and D. P. Atherton, "Obtaining controller parameters for a new Smith predictor using autotuning," Automatica, vol. 36, no. 11, pp. 1651-1658, 2000.

[14] J. E. Normey-Rico and E. F. Camacho, "Dead-time compensators: a survey," Control Engineering Practice, vol. 16, no. 4, pp. 407-428, 2008.

[15] T. Liu and F. R. Gao, "Enhanced IMC design of load disturbance rejection for integrating and unstable processes with slow dynamics," ISA Transactions, vol. 50, no. 2, pp. 239-248, 2011.

[16] E.-D. Cong, M.-H. Hu, S.-T. Tu, F.-Z. Xuan, and H.-H. Shao, "A novel double loop control model design for chemical unstable processes," ISA Transactions, vol. 53, no. 2, pp. 497-507, 2014.

[17] J. E. Normey-Rico, P. Garcia, and A. Gonzalez, "Robust stability analysis of filtered Smith predictor for time-varying delay processes," Journal of Process Control, vol. 22, no. 10, pp. 19751984, 2012.

[18] J. Nandong and Z. Zang, "High-performance multi-scale control scheme for stable, integrating and unstable time-delay processes," Journal of Process Control, vol. 23, no. 10, pp. 13331343, 2013.

[19] A. S. Rao and M. Chidambaram, "Analytical design of modified Smith predictor in a two-degrees-of-freedom control scheme for second order unstable processes with time delay," ISA Transactions, vol. 47, no. 4, pp. 407-419, 2008.

[20] T. Liu, W. Zhang, and D. Gu, "Analytical design of two-degreeof-freedom control scheme for open-loop unstable processes with time delay," Journal of Process Control, vol. 15, no. 5, pp. 559-572, 2005.

[21] P. García and P. Albertos, "Robust tuning of a generalized predictor-based controller for integrating and unstable systems with long time-delay," Journal of Process Control, vol. 23, no. 8, pp. 1205-1216, 2013.

[22] V. Vijayan and R. C. Panda, "Design of PID controllers in double feedback loops for SISO systems with set-point filters," ISA Transactions, vol. 51, no. 4, pp. 514-521, 2012.

[23] P. García, P. Albertos, and T. Hägglund, "Control of unstable non-minimum-phase delayed systems," Journal of Process Control, vol. 16, no. 10, pp. 1099-1111, 2006.

[24] P. Albertos and P. García, "Robust control design for long timedelay systems," Journal of Process Control, vol. 19, no. 10, pp. 1640-1648, 2009.

[25] J. C. Moreno, J. L. Guzmán, J. E. Normey-Rico, A. Baños, and M. Berenguel, "A combined FSP and reset control approach to improve the set-point tracking task of dead-time processes," Control Engineering Practice, vol. 21, no. 4, pp. 351-359, 2013.

[26] C. Dey, R. K. Mudi, and D. Simhachalam, "A simple nonlinear PD controller for integrating processes," ISA Transactions, vol. 53, no. 1, pp. 162-172, 2014. 
[27] J. E. Normey-Rico and E. F. Camacho, "Unified approach for robust dead-time compensator design," Journal of Process Control, vol. 21, no. 7, pp. 1080-1091, 2011.

[28] K. J. Astrom and H. Hagglund, PID Controllers: Theory, Design and Tuning, Instrument Society of America, Research Triangle Park, NC, USA, 2nd edition, 1995.

[29] B. A. Ogunnaike and W. H. Ray, Process Modeling, Dynamics and Control, Oxford University, New York, NY, USA, 1994.

[30] M. Shamsuzzoha and M. Lee, "Enhanced performance for twodegree-of-freedom control scheme for second order unstable processes with time delay," in Proceedings of the International Conference on Control, Automation and Systems (ICCAS '07), pp. 240-245, Seoul, Republic of Korea, October 2007.

[31] M. Morari and E. Zafiriou, Robust Process Control, Prentice Hall, Englewood Cliffs, NJ, USA, 1989.

[32] J. C. Doyle, B. A. Francis, and A. R. Tannenbaum, Feedback Control Theory, Macmillan Publishing Company, New York, NY, USA, 1992. 


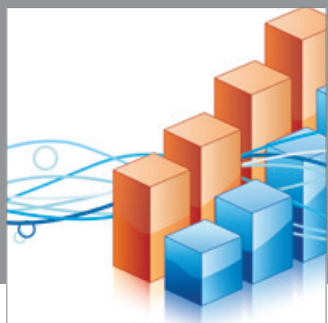

Advances in

Operations Research

mansans

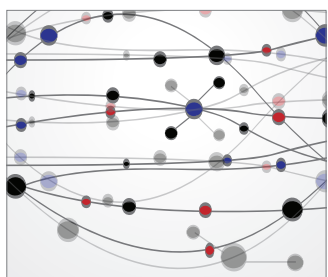

The Scientific World Journal
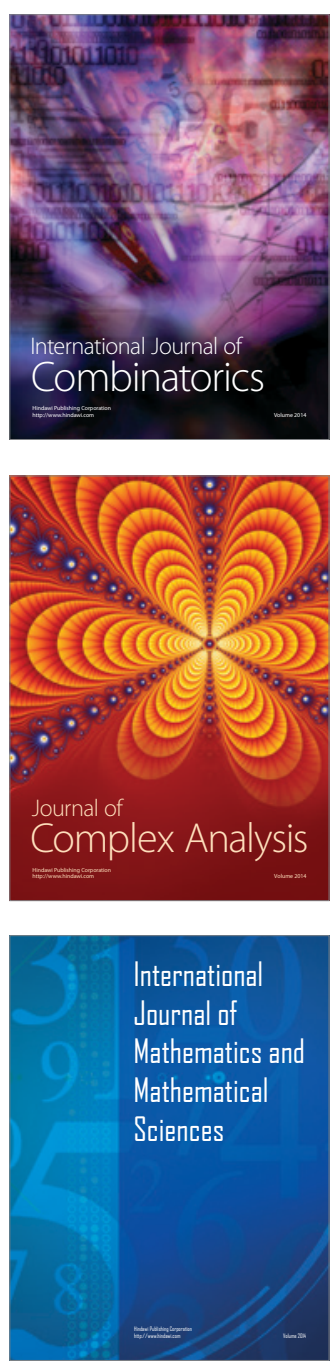
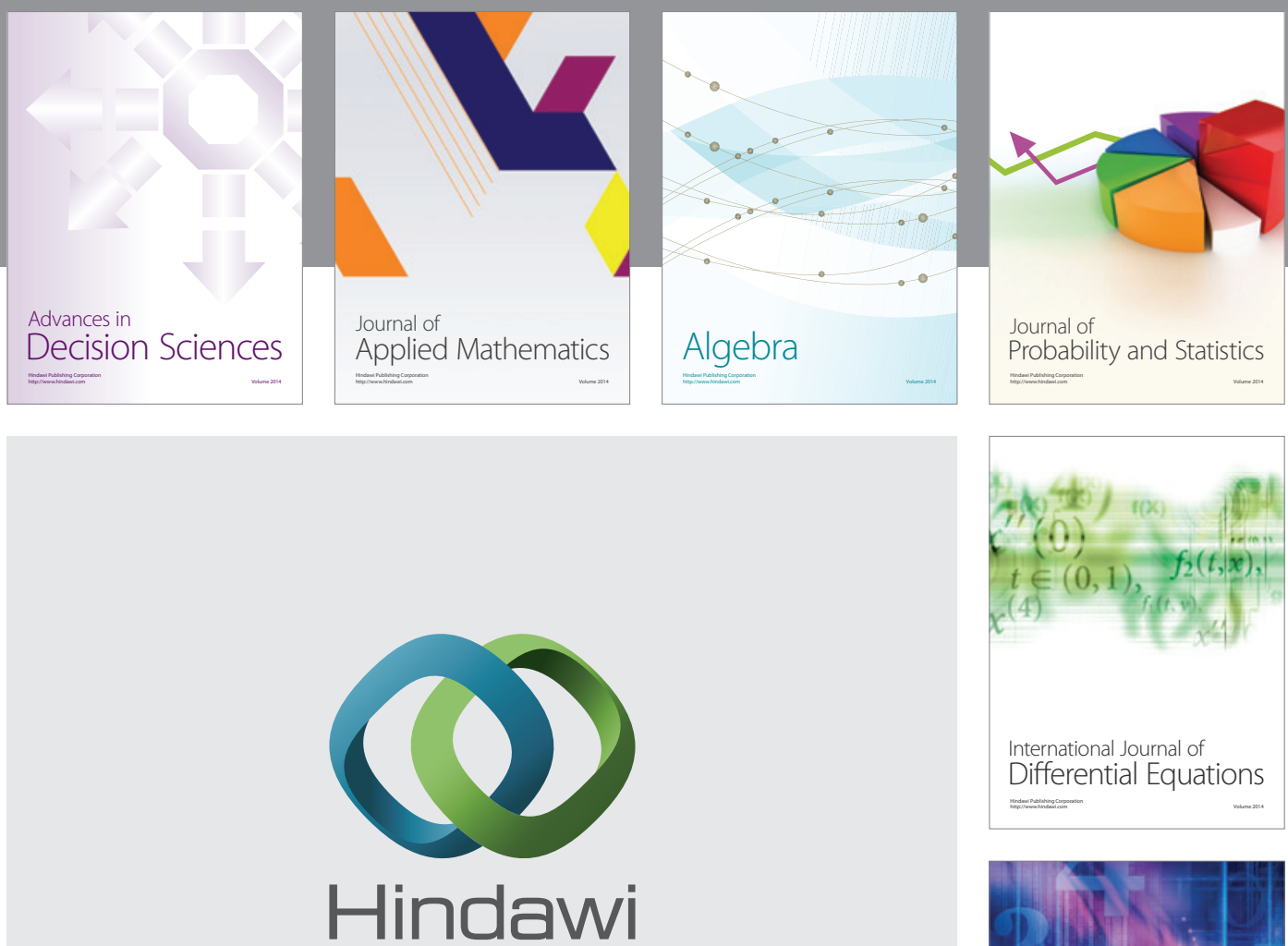

Submit your manuscripts at http://www.hindawi.com
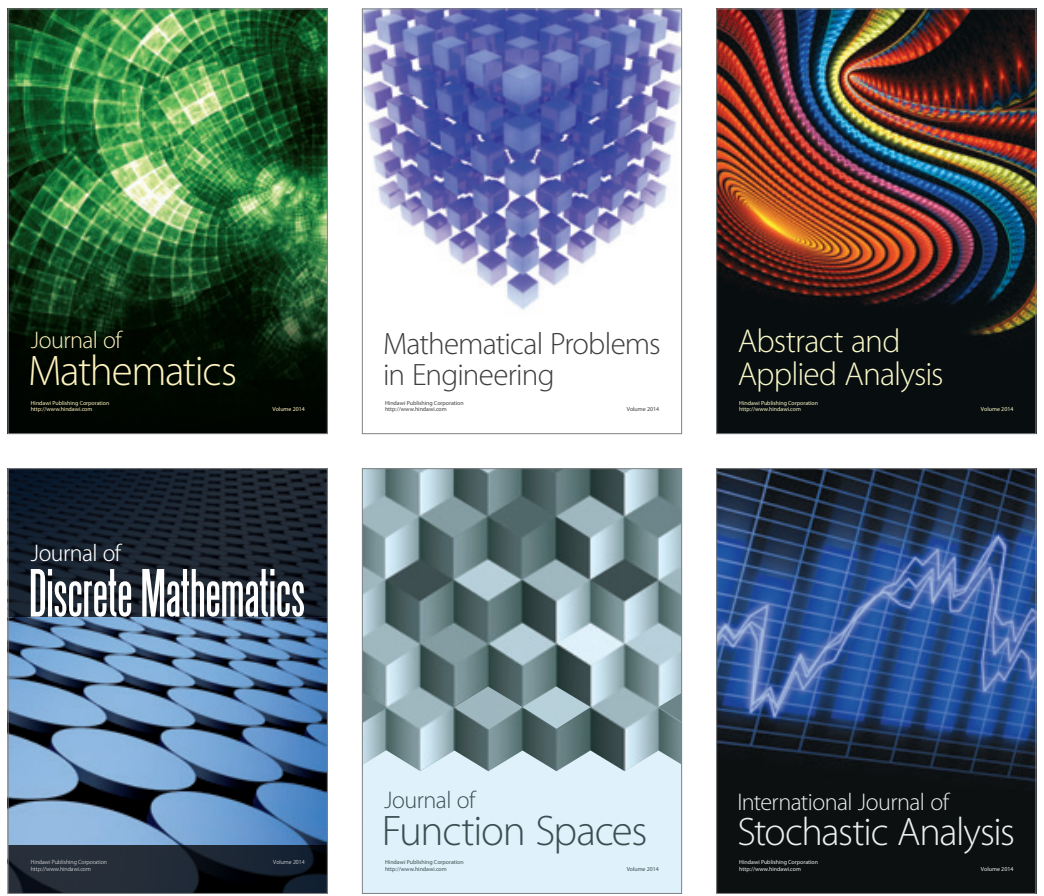

Journal of

Function Spaces

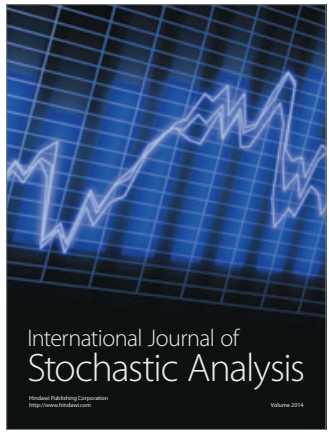

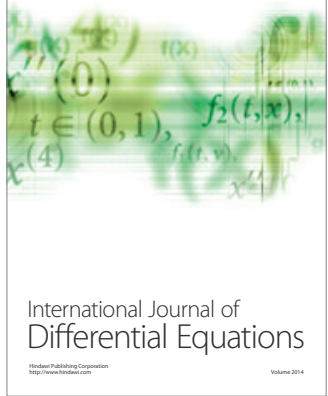
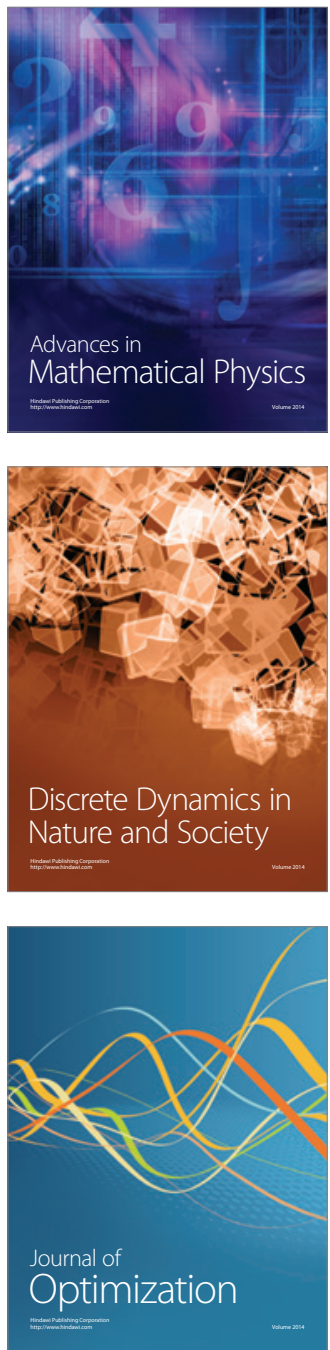